\title{
Modelling daily activity program generation considering within-day and day- to-day dynamics in activity-travel behaviour
}

\section{KHANDKER M. N. HABIB ${ }^{1} \&$ ERIC J. MILLER ${ }^{2}$}

${ }^{1}$ Post Doctoral Fellow, Department of Civil Engineering, University of Toronto, 35 St George Street, Toronto, Ontario M4S 1A4, Canada, Phone: 416978 5049, Fax: 416978 5054, khandker.nurulhabib@utoronto.ca

${ }^{2}$ Bahen-Tanenbaum Professor, Department of Civil Engineering, Director of Joint Program in Transportation, University of Toronto, 35 St George Street, Toronto, Ontario M4S 1A4, Canada, Phone: 416978 4076, Fax: 416978 5054, miller@ecf.utoronto.ca

\section{Citation:}

Habib, K.M.N., Miller, E.J. 2008. Modelling daily activity program generation considering within-day and day-to-day dynamics in activity-travel behaviour. Transportation 35:467484

DOI 10.1007/s11116-008-9166-8 
Abstract: This paper presents Kuhn-Tucker demand system models for daily activity program generation. The models are for day-specific activity program generations of a week-long modelling span. The models accommodate within-day and day-to-day dynamics in time-use and activity-travel behaviour explicitly. The activity types considered are the non-skeletal and flexible activities. These activities are divided into 15 generic categories. Under the daily time budget and non-negativity of participation rate constraints, the models predict the optimal set of activities (given the average duration of each activity type). The daily time budget considers the at-home basic needs and night sleep activities as a composite activity. The concept of composite activity ensures the behavioural dimension of time allocation and activity/travel behaviour in a sense that the activities corresponding to the composite activity are regular skeletal activities but highly flexible in nature. We are sure to execute these activities but do not often allocate precisely a specific amount of time to them during advanced planning. Workers' total working hours (skeletal activity and not a part of the time budget) are considered as a variable in the models to accommodate the scheduling effects inside the generation model. The incorporation of previous day's total executed activities as variables introduces day-to-day dynamics into the activity program generation models. The possibility of zero frequency of any specific activity under consideration is ensured by the Kuhn-Tucker optimality condition used. The models use the concept of goal/direct utility of activity episodes. The empirical estimations of the models are done using 2002-2003 CHASE survey data collected in Toronto. The models perform well in terms of fitting the observed data.

Key words: activity generation, day-to-day dynamics, within-day dynamics, travel behaviour, activity utility 


\section{Introduction}

The dramatic paradigm shifts from trip-based to activity-based analysis of travel behaviour in recent years is land-marked by several operational activity-based travel demand models. ALBATROSS, CEMDAP, FAMOS, and TASHA are most cited in the literature as operational activity-based travel demand models (Arentze \& Timmermans 2005; Bhat et al. 2004; Pendyala et al. 2004; Miller \& Roorda 2003). All of these models can be divided into two major parts: activity program generation and activity scheduling (Bhat \& Koppelman 1993; Habib \& Miller 2006b 2006c). Another common feature is the modelling span, which is a 'typical day'. Two important, practical issues associated with this state of the art are the modelling time-span and the procedures used to generate the activity program.

Considering a typical day as the modelled time-span has become a commonplace in activity-travel demand modelling. A typical day assumption refers to a hypothetical day to overcome the day-specific features of a week. The argument is that it reduces the data requirements for model development and also simplifies the computational issues. But our activity-travel behaviour shows significant variability across the week (Kitamura et al. 2003; Schlich \& Axhausen 2003; Doherty et al. 2004). Neglecting these daily variations undermines the basic principle of the paradigm shift to the activity analysis: that travel is a derived demand. The derived demand concept indicates the evolution of activity-travel pattern over time (Doherty 2006). It is obvious that the day-to-day variations are intricately related to the within-day variations of the activity-travel behaviour too. Modelling within-day variations in activity-travel behaviour in a typical day model thus becomes incomplete or adhoc if the day-to-day variations are not addressed properly. 
On the other hand, the modelling techniques for the activity program generation in all operational models use either empirical distributions or a decision tree approach for generating activity episode frequency, duration, etc. The empirical distribution approach (Pendyala et al. 2004; Miller \& Roorda 2003) uses observed distributions of different activity attributes to simulate activity generation. It considers the distributions to be constant over time. This approach requires some index variables to identify different market segments. Unless the relationship between index variables and the objective variables of concern are econometrically justified, this can lead to spurious results (Habib \& Miller 2006a). The decision-tree approach develops conditional decision structure about different activity attributes to simulate activity generation (Arentze \& Timmermans 2005). In this case an exhaustive set of possible condition variables is defined for the homogeneous condition state and based on this the corresponding decision trees for the decision variables are devised. This approach can also lead to inaccurate/spurious relationships or 'relationships-by-chance' if the condition variables do not have econometric relationship with the decision variables (Habib \& Miller 2006c).

This paper addresses these issues of modelling time-span and activity program generation process. It presents econometric models of activity program generation. The target is the nonskeletal activities that are temporally and spatially more flexible, unlike skeletal activities. The skeletal activities refer to the activity types that are parts of medium- to long-term decision processes, e.g. work/school activities. In case of skeletal activities the activity planning process does not circulate within short time (daily or weekly basis). Whereas non-skeletal activities are often planned on a short-term basis and more adjustable than skeletal activities. Also, the possibilities of repeated participation or non-participation in some specific activity types are inherent in the models that allow capturing the underlying weekly dynamics in time-use and the 
activity-travel behaviour. For the explicit consideration of within-day dynamics in time-use behaviour, the worker's total number of working hours (skeleton activity) is considered as a variable in the models and for the day-to-day dynamics of time-use and activity-travel behaviour, the previous day's total executed activities are considered as variables in the models. The models are econometric in nature and exploit the concept of activity utility. The models represent the large scale demand system modelling approach, where the demand of all activities of concerns are modelled simultaneously and the possibility of non-participation in any specific activity is ensured by adopting Kuhn-Tucker optimality conditions. The empirical estimation of the models uses 2002-2003 CHASE (Computerized Household Activity Scheduling Elicitor) survey data collected in Toronto, Canada.

The rest of the paper is organized as follows: the next section discusses the activity program generation or activity agenda formation process in details, followed by the sections discussing the concept of activity utility, econometric specification and estimation procedures of the models, description of the data and the interpretations of the estimated models. The paper concludes with a summary of the key findings.

\section{Activity program generation}

The activity program generation stage of an activity-based model generates a set of different activities that are to be scheduled within a specific time frame. A generic set of 'things to do' within a planned period of time that does not necessarily involve a scheduled sequence is called an activity-agenda (Litwin 2005). Conceptually it represents the continuous mental process, where different short-, medium- and long-term household decisions interact with each other. Practically it is difficult to observe the individual's mental processes. We really do not observe 
the activity agenda formation process. We can best observe the collection of different types of activities of a particular individual prior to the scheduling process. Though the behavioural process of activity program formation is difficult to capture, we can observe the outcome of the process and make logical hypotheses to simulate the behavioural processes (Habib \& Miller 2006a). The state-of-the-art activity data collection process CHASE can collect activity-travel information prior to the scheduling process (Doherty et al. 2004; Doherty 2006; Doherty 2000).

The hypothesis in this paper is that, for a particular person, the collection of the activity episodes (activities with duration and other attributes) that are to be scheduled within a specific time period is the optimum set among a number of possible sets he/she desires to schedule. The challenge is to simulate the behavioural process that gives this observed outcome. All of the operational activity-based travel demand models lack the explicit definition of this activity program formation in terms of its behavioural process and multiple temporal dimensions (Litwin 2005). While all models accommodate conceptually a similar level (activity program generation) of decision-making process, the lack of an explicit definition and incorporation of behaviourally plausible hypotheses may influence the policy sensitivities of the models. This is also important from the model integration points of view. In case of an integrated land-use and transportation model, it can provide sufficient scope to connect different levels of the decision-making process (short-, medium- and long-term) (Habib \& Miller 2006a; Habib \& Miller 2005). On the other hand, the proper interface of the activity-travel demand model with the other components of an integrated land-use and transportation model requires an appropriate technique that accommodates necessary means for proper linkage (Salvini \& Miller 2005). The concept of utility provides the opportunity to accomplish this goal. Considering the multidimensional nature of interactions and trade-offs in our daily life, it is virtually inevitable that a utility-based 
measure is necessary to build an operational activity-based travel demand model (Miller 2005). The next section discusses the concept of activity utility used to derive the specification of the models.

\section{Concept of activity utility and specification of the model}

We use the utility concept of activity proposed by Winston (1987). The utility of an activity is divided into two parts: the goal utility and the process utility. The goal utility is gained by the end state accomplished by the activity and the process utility is gained by the activity execution process per se. The goal utility component represents the direct utility of the activity episodes and defines the activity program generation stage. Thus this utility component can be a function of activity episode duration but at the moment of activity program generation stage, the duration need not be based on an explicit utility-based calculation (Miller 2005). On the other hand, the process utility is a feature of activity itself and will generally depend upon duration, and the actual episode duration will depend on activity scheduling and rescheduling trade-offs for different candidate activities drawn from the activity-agenda. So, for modelling the activity program generation, the goal utility of activity episode is important, whereas the process utility component may primarily affect activity scheduling and rescheduling trade-offs. We use the specification of the goal utility used by Habib \& Miller (2006a) as originally proposed by von Haefen \& Phaneuf (2004). Habib and Miller (2006a) investigated the feasibility of utility theory to model activity-agenda formation and tested different alternative model specification considering a whole week as modelling span. As an extension of that work, this paper investigates the dynamics in activity-agenda formation across the week. In this specification the total utility of the activity program (activity-agenda) is divided into two parts: the utility derived 
from all activities under consideration and the utility derived from all other unmodelled activities. The unmodelled activities can be referred as composite activity. The term composite activity indicates the activity types that are not of direct interest within the model but most still be accounted for in the aggregate since we do need to account for composite time allocation within the overall time budget. Under limited time budget this activity indicates the leftover time (Z) after planning for the specific activities under consideration. Composite activity may be composed of more than one activity type, but with respect to the specific activity types under consideration, these are not quality differentiable and hence can be considered together. The total time budget $(T)$ for the activity agenda is divided between a bundle of all specific activities and the composite activity simultaneously. The trade-off among the specific activities within the bundle is also embedded within the specification. The utility function for the specific activities $\left(U_{j}\right)$ has two components: the baseline utility and the additional utility. The baseline utility fraction $\left(U_{\text {baseline }}\right)$ is the exponential function that refers to the baseline preference for a specific activity compared to the composite activity and the additional utility defines the quantitative consumption of the specific activity. The additional utility fraction $\left(U_{\text {additional }}\right)$ is considered to have a logarithmic functional form to ensure a decreasing rate of satisfaction with increasing consumption. It also contains a constant term, the translating parameter $(\theta)$ that ensures the possibility of zero consumption. The translating parameter provides the reference to allow for the planning of zero frequency of any specific activity episode under consideration, which refers to the zero consumption option. Under the assumption of Kuhn-Tucker optimality conditions, zero consumption is defined by a corner solution. So, the specification of the total utility of an activity agenda becomes: 


$$
\begin{aligned}
U_{\text {total }} & =\sum_{j=1}^{N} U_{j}+U_{Z} \\
& =\sum_{j=1}^{N}\left(U_{\text {Baseline }} * U_{\text {additional }}\right)_{j}+U_{Z} \\
& =\sum_{j=1}^{N} e^{\left(\left(\beta^{p} X^{p}\right)+e^{\delta} \varepsilon_{j}\right)} * \ln \left(e^{\left(\beta^{a} X_{j}{ }^{a}\right)} Y_{j}+e^{\theta}\right)+\frac{1}{1-\exp (\rho)} z^{(1-\exp (\rho))}
\end{aligned}
$$

Where,

$U_{j}$ is the utility of specific activity episode

$U_{\text {baseline }}$ is the baseline utility fraction of the utility of specific activity episode

$U_{\text {additional }}$ is the additional utility fraction of the utility of specific activity episode

$U_{z}$ is the utility of the composite activity

$X^{p}$ is the vector of variables defining the person's tastes and preferences

$\beta^{p}$ is the parameter corresponding to $X^{p}$

$\delta$ is the scale parameter

$X^{a}$ is the vector of variables corresponding to the attraction/aversion to activity $j$

$\beta^{a}$ is the parameter corresponding to $X^{a}$

$Y_{j}$ is the frequency of the activity $j$ (the quantitative consumption)

$N$ is the total number of specific activities under consideration

$\theta$ is the translating parameter that defines the slope of the indifference curve and ensures the feasibility of corner solutions.

$\varepsilon_{j}$ refers to the activity specific idiosyncratic term

$Z$ is the time allocated to the composite activity

$\rho$ is composite activity parameter that defines the importance of composite activity in activity program formation process 
This specification ensures the overall utility function of the activity-agenda is a strictly increasing function of each individual activity type and the composite activity enters as additive to the collective sub-function of individual activities. It ensures the scope of modelling the tradeoff between planned and unplanned activities (composite activity) at the first stage. This specification also ensures that if an activity is not chosen (i.e. $Y_{j}=0$ ) the changes in the corresponding $X_{a}$ variables do not have any influence on the total utility of the activity program (activity-agenda). That means the derivative of the total utility function with respect to any attractor variable $X_{a}$ of any particular activity is zero if the corresponding frequency $Y_{j}$ is zero. This property indicates that the addition of any activity type in the choice set of activity-agenda does not have any effect on the agenda utility unless that specific activity is chosen. Thus it allows considering very large choice sets in the demand system, which is advantageous for daily activity program generation modelling, where we can specify numerous types of activities for consideration. Another key point is that this functional form is a direct utility function with an endogenous idiosyncratic error component that gives rise to an endogenous regime switching type of model under time budget and non-negativity of activity frequency constraints. The functional form is strictly differentiable, strictly increasing and quasi-concave, which can give $2^{N}$ combinations of possible activity-agenda sets, where $N$ is the total number of activities in the choice set (2 represents 1 for interior and 1 for corner solution).

Now considering a specific time budget, the proposed utility maximization model for activity-agenda formation becomes: 


\section{Maximize:}

$U\left(X^{p}, X^{a}, \varepsilon\right)=\sum_{j=1}^{N} e^{\left(\left(\beta^{p} X^{p}\right)+e^{\delta} \varepsilon_{j}\right)} \ln \left(e^{\left(\beta^{a} X_{j}{ }^{a}\right)} Y_{j}+e^{\theta}\right)+\frac{1}{1-\exp (\rho)} z^{(1 \exp (\rho))}$

Subject to:

$$
\begin{aligned}
& \sum_{j} d_{j}{ }^{T} \cdot Y_{j}+z=T, \quad d_{j} \text { indicates the average duration of activity } j \\
& T \text { indicates the total time budget }
\end{aligned}
$$

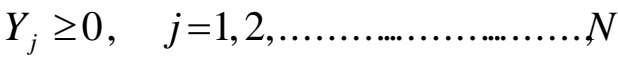

$$
\begin{aligned}
& z \geq 0
\end{aligned}
$$

To ensure the possibility of corner solutions we exploit the Kuhn-Tucker optimality condition.

For this optimization problem the Kuhn-Tucker first order optimality conditions are:

$$
\begin{aligned}
& \frac{\partial U}{\partial Y_{j}}-d_{j} * \lambda \leq 0 \\
& \& \\
& \frac{\partial U}{\partial z}-\lambda \leq 0
\end{aligned}
$$

Where

$\lambda$ is the Lagrange Multiplier.

The equality condition of equation (2) holds when the $Y_{j}$ is non-zero. Considering the equality for $z$, the constant $\lambda$ can be expressed as:

$$
\lambda=\frac{\partial U}{\partial z}
$$

Substituting the value of $\lambda$, we get:

$$
\frac{\partial U}{\partial Y_{j}} \leq d_{j} * \frac{\partial U}{\partial z} \quad j=1,2,3, \ldots \ldots \ldots \ldots . . N
$$




$$
\begin{aligned}
\varepsilon_{j} \leq f_{j}\left(X^{p}, \beta^{p}, \delta, X^{a}, \beta^{a}, Y_{j}, \theta, \rho\right): & \\
\varepsilon_{j}=f_{j}\left(X^{p}, \beta^{p}, \delta, X^{a}, \beta^{a}, Y_{j}, \theta, \rho\right) & \text { if } Y_{j}>0 \\
\varepsilon_{j}<f_{j}\left(X^{p}, \beta^{p}, \delta, X^{a}, \beta^{a}, Y_{j}, \theta, \rho\right) & \text { if } Y_{j}=0
\end{aligned}
$$

Putting the exact expressions of the total utility function, we get:

$$
\varepsilon_{j} \leq \frac{1}{\exp (\delta)}\left(\begin{array}{r}
-\left(\exp \left(\left(\beta_{0}+\beta^{P} X^{P}\right)\right)+\right. \\
+\ln \frac{d_{j}}{\exp \left(\beta^{a} X^{a}\right)} \\
+\ln \left(\exp \left(\beta^{a} X^{a}\right) * Y_{j}+\exp (\theta)\right) \\
+\left(-\exp (\rho) \ln \left(T-\sum_{j} d_{j} Y_{j}\right)\right)
\end{array}\right), \forall j
$$

Intuitively the above conditions indicate that for positive-frequency activities the marginal utilities are the same across the optimal allocation. Assuming a distributional assumption of $\varepsilon_{j}$, we can easily derive the likelihood function for estimating the structural parameters of the proposed model. Let us consider an individual, $i$ with the activity frequencies $Y_{j}>0$ for $j \leq K$ and $Y_{j}=0$ for $j>K$ (where $j=1,2,3 \ldots N$ and $K$ is the total number of non-zero activity frequencies). Using the 'transformation of variable theorem', the contribution of this person $i$ to the overall likelihood function of the sample is:

$$
l=\int_{-\infty}^{f_{K+1}} \ldots \ldots \ldots \ldots . . \int_{-\infty}^{f_{N}} G\left(f_{1}, f_{2}, \ldots \ldots . . f_{K}, \varepsilon_{K+1}, \ldots \ldots \varepsilon_{N}\right)|J| d \varepsilon_{K+1}, \ldots . . d \varepsilon_{N}
$$

Where,

$G($. ) indicates a generating function.

$|\mathrm{J}|$ is the determinant of the Jacobian of transformation from $\left(\varepsilon_{1}, \varepsilon_{2}, \varepsilon_{3}, \ldots \ldots \ldots \varepsilon_{N}\right)$ to $\left(Y_{1}, Y_{2}\right.$,

$Y_{3}, \ldots \ldots \ldots \ldots \ldots Y_{K}, \varepsilon_{K+1}, \ldots \varepsilon_{N)}$. 
Here the Jacobian, $\mathrm{J}=\partial \varepsilon_{j} / \partial S_{j}$, where $S_{j}=Y_{K}$ if $j \leq K$ otherwise $S_{j}=\varepsilon_{K}$. This gives the Jacobian a partitioned structure whose lower right component is identity and the upper right component is zero. So, the determinant of the Jacobian becomes just the determinant of a $K \mathrm{X} K$ matrix of $\partial \varepsilon_{j} / \partial Y_{j}$

Considering that the $\varepsilon_{j}$ has a normalized type I extreme value (IID) distribution, we get the likelihood function:

$l\left(Y \mid \beta_{0}, \beta^{a}, \delta, \beta^{p}, \theta, \rho\right)=\prod_{j}\left(\left[\exp \left(-f_{j}(.)\right) / \exp (\delta)\right]^{1_{Y_{j}>0}}|J| \exp \left[-\exp \left(-f_{j}().\right)\right]\right)$

Where

$1_{Y j>0}$ is the indicator function for the chosen activity

So, if the total number of individual observations is $P$ in the sample, the total likelihood function becomes

$L\left(Y \mid \beta_{0}, \beta^{a}, \delta, \beta^{p}, \theta, \rho\right)=\prod_{P}\left(\prod_{j}\left([\exp (-f(.)) / \exp (\delta)]^{1_{Y_{j}>0}} \cdot|J| \cdot \exp [-\exp (-f())].\right)\right)$

This is a closed form likelihood function. For a fixed parameter model, the structural parameters of the above likelihood function can be estimated by using a numerical gradientbased search method for fairly large number of activities without any difficulties. For this paper, the loglikelihood function is estimated using the code written in GAUSS using BFGS algorithm (Aptech Systems, 2006; von Haefen RH and Phaneuf DJ, 2004).

Once the structural parameters of the model are estimated the model becomes simply a constrained optimization problem. This means for given structural parameters the optimization 
technique should be used to estimate optimum values of activity frequencies $\left(Y_{j}\right)$ and composite activity time $(Z)$. To obtain the optimum activity-agenda of an individual, the objective function should be integrated over the possible values of unknown error terms (a similar approach is taken by Bhat, 2005). In other words, being consistent with the behavioural assumption that people are not always global optimizers, one can generate a set of random numbers as a candidate vector for the unknown error terms in the model and plug those values in the objective function to generate a random activity-agenda for that person (Habib \& Miller 2006a). However, it should be mentioned that this optimality does not refer to the global optimization of activity behaviour that is strongly denied by behavioural psychologists (Gärling et al. 1989; Gärling \& Garvill 1993;

Gärling et al. 1994). This refers to the optimality of a sub-module of overall activity decision framework that is behaviourally plausible (Habib \& Miller 2006a).

\section{Data and Variable Specification}

CHASE survey data from the first wave of the Toronto Travel-Activity Panel survey (TAPS) are used in this paper. The survey was conducted in Toronto, in 2002-2003 with 426 individuals in 271 households. The detail description of the methods and general data summary are available in Doherty et al. (2004) and Doherty \& Miller (2000). This is a multi-day self-reporting survey. The participant in this survey lists the processes of activity planning and scheduling over a period of 7 days. The participant first adds the activities before starting of the day and may or may not modify before the activities are performed. The CHASE program tracks activity episodes that are added first, then modified or deleted over time. The implementation state of an activity episode in terms that whether the episode got modified before implementation is also available. The 'added' attributes of the episode indicate the first entry and are thereby assumed to be the output 
of the mental process of activity program generation. To simulate the activity program generation process, the choice set of the activity-agenda is defined by individual activity types. The generic activity classification is devised to ensure the homogeneity in terms of basic type and average duration of the activities within the same group. The basic activity types are:

1. Act01: Basic needs like lunch, coffee etc but not at home

2. Act02: Household obligations: cleaning, maintenance, meal preparation, attending kids, attending pets etc.

3. Act03: Drop off / Pick up goods like video/CD/DVD rental, dry cleaning, postal mails etc.

4. Act04: Shopping like Major grocery shopping (at least 10 items)

5. Act05: Shopping like Minor grocery shopping (less than 10 items), convenience store, drug store etc.

6. Act06: Shopping like Personal clothing, houseware etc.

7. Act07: Services like doctors / medical appointment etc.

8. Act08: Services like saloon/barber/beauty, banking, auto servicing etc.

9. Act09: Recreations like hobbies, workout, sports, theatre, other outdoor recreational activities

10. Act10: Recreations like at home TV program, video, napping, relaxing etc.

11. Act11: Social activities like visiting/hosting, planned social events, bars/special clubs etc.

12. Act12: Social activities like religious and cultural activities

13. Act13: Other all types of activities that fall as both social as well as recreational type activities but do not belong to above types

14. Act14: ICT use like telephone (more than 10 minutes), internet shopping, browsing etc.

15. Act15: Volunteer activities 
It is clear that the above-mentioned activities do not include work/school, night sleep and athome basic need type activities because these are considered skeletal (most regular) activities and are to be modelled separately (Habib \& Miller 2006c). It should be mentioned that among the skeletal activities work/school is considered a fixed type activity that is often defined by external factors (job type, office schedule) and hence is deducted from the time budget it his model. On the other hand, night sleep and at home basic type activities are quite regular (skeletal) but highly flexible too, hence these are considered as the composite activity that provides the slackness in daily time allocations.

A typical week is considered for the model time-span. Monday (Day0) is taken as the start of the week. Saturday (Day5) and Sunday (Day6) are the weekends. After cleaning for missing information, a total 405 out of 426 individuals remain in the data set for model estimation. To give some idea of the composition of weekly activities by day, Figure 1 presents the maximum daily frequencies of the activities in observed in the data set. Considerable variations are clear across the week. For Act01 the maximum frequency is the highest at the middle of the week, for Act02 the maximum frequency is the highest for the first day of the week and the last day of the weekends. Act03, Act04 and Act07 show constant maximum frequency across the week. Considerable variations in non-major grocery shopping (Act05 and Act06) are also clear in the data. Other than the maximum frequency, the data reveal that people participate in some specific type of activities repeatedly throughout the week whereas lower participation rates are also clear for some activities (serial non-participation). These results provide some assurance that the sample represents a typical week of the individuals under consideration. 
For modelling the activity program generation process, the time budget $(T)$ for each day is considered as 24 hours and for whole week time budget it is 7 times 24 hours. The average duration $\left(d_{j}\right)$ of the activities are considered to be the weekly average values. Each individual is considered to have a choice set consisting of the 15 activity types described above. Several socio-economic variables are also considered as covariates $\left(X^{p}\right)$. These are: the individual's age, gender, total yearly income in Canadian Dollars, an indicator of having driving license, total work/school hours in the week, size of the person's household and total number of autos in the household. Two activity-specific variables $\left(X^{a}\right)$ are considered: the 'number of possible activity locations' and 'travel ratio'. The travel ratio is the ratio of the summation of activity duration and travel time to the activity duration (Ettema 2005). It refers to the price of activity time in terms of travel time. By definition it is greater than or equal to 1 . The longer the travel time required to get to an activity location, the higher the value of the 'travel ratio'. This variable brings trip information inside the activity program generation model in a normalized way, rather than simply using travel time. This variable makes the model sensitive to transportation system performance. The 'number of possible locations' variable represents the spatial distribution of activity spaces for the person. This variable is collected during the survey by the EWR (End of Week Review) questions. This is also a key variable that defines the spatial dispersion of activity places and its influence on activity/travel behaviour. This variable makes the model sensitive to various land-use policies.

Worker's total number of daily working hours (skeletal activity time) is considered as a variable for the baseline utility component of day-specific models. For the week-long model, this variable is the total weekly working hours. This variable connects the skeletal versus nonskeletal activity planning. Work is considered as skeletal activity and the assumption is that 
planning of such activity is the part of medium- to long-term decision processes. For short-term activity planning (daily or weekly) this activity type is not considered as part of activity-program formation. But considering the total time allocation to this skeletal activity in the non-skeletal activity-agenda formation model ensures the interactions between these two basic activity types and helps to address the within-day dynamics if time allocation and activity planning behaviour. Assuming the weekly cycle as the rhythm of life, day-to-day variations and trade-offs are obvious in our activity/travel behaviour. The issues of repeated participation and serial nonparticipation in specific activities are also clear in the data. In order to accommodate these issues another variable is considered in the model: the previous day's total executed activity frequencies. This variable is included in the additional utility component; so that the previous day's total executed frequencies will influence the present day's activity program formation. This variable accommodates the day-to-day variations in activity program formation and it also introduces an activity scheduling element inside the activity generation process.

\section{Interpretations of the empirical models}

Models are developed for each day of the week, considering Monday as the starting day. For comparison purposes, a weekly model is also developed, considering whole week as the planning period. The models are presented in Table 1 . Considering the relatively small data set compared to the large number of parameters to be estimated, the estimated coefficients are considered statistically significant if the corresponding two-tailed ' $t$ ' statistics satisfy the $90 \%$ confidence interval, $(\mathrm{t} \geq 1.64)$. However, the variables with statistically insignificant parameters are also retained in the models because they provide considerable insight into the behavioural process and also due to the assumption that if a larger data set were available these parameters might 
show statistical significance. The goodness of fit of the overall model is measured by the pseudo$\mathrm{R}$ square value, which is 1 minus the ratio of log-likelihood value of the full model and the loglikelihood value of the null model (constant only model). The pseudo-R square value closer to 1 represents better fit to the observed data.

Individually, the goodness of fit values of the day-specific models is better than the aggregate weekly model. This is because of the within-day and day-to-day variations in time-use and activity/travel behaviour. The aggregate weekly model suppresses these variations, whereas in the day-specific models these are addressed explicitly. Among the day-specific models, the Monday model, which represents the beginning of the week, gives lower goodness of fit value. This is because this model does not consider the variable 'previous day's total executed activities'. The CHASE data used in this paper considered Monday as the beginning of the week and the data collection started on Monday. Hence the data are left censored for Monday per se and we lack the information of previous day's executed activities. This raises the issues of rhythm of life: 'what should be the appropriate planning span?' Although a multi-week travel diary survey reveals that travel behavior is neither totally repetitious nor totally variable, a multiyear panel survey of activity/travel behaviour justifies a typical week as a minimum unit of rhythm of life (Roorda \& Ruitz 2006). The best goodness of fit is seen for Thursday model, which is the middle of the week. Also the maximum number of statistical significant parameters is for the Thursday model. As, Thursday is the middle of the week, the modelling approach considering previous day's executed activities together with dynamic linkage between skeletal and non-skeletal activity information enables to capture maximum behavioural elements. This is also indicating that for a single-day modelling many of the behavioural elements remain censored. 
In terms of the components of individual models, the socio-economic variables in the baseline utility components are considered generic because this reduces the number of parameters to be estimated. At the same time it is justified in the way that the baseline utility component mainly defines the trade-offs in time allocation to specific activities and the composite activity but the activity specific dummy variables in the baseline utility component introduce the individual activities under consideration explicitly. So, the socio-economic variables in the baseline utility component in general indicate the activity participation tendencies and the activity-specific dummy variables indicate the baseline relative importance of individual activities in activity program generation. The variables in the additional utility component indicate individual activity's potential/attraction to derive the optimum set of activities. The translating parameter $\theta$ gives the reference for the additional utility of zero frequency activities and the composite activity parameter $(\rho)$ indicates the effects of the time budget in activity program formation. For a coherent preference specification, the value of 1$\exp (\rho)$ should be less than +1 . A lower value of $1-\exp (\rho)$ indicates the lower effect of time budget or the composite activity on the activity program generation.

The specification of the models is in general a demand system model specification, where under a given time budget constraint and the given average duration of the activities, the models determine whether to participate (discrete) in an activity and, if participating, then how many times (continuous) to participate. In such a specification the scale parameter of the extreme value error plays a key role in linking the discrete and continuous part of the model (Arora et al. 1998) and also according to the equation (1), the higher the value of the scale parameter (exponential of the parameter), the higher the randomness in activity program generation. According to Table 1, the randomness in activity program generation is the highest for Sunday, which is consistent with 
our expectation that weekend activities are more random per se than weekday activities. Comparing the day-specific models and the weeklong model, the randomness reduces when the planning period is the whole week and behaviourally it indicates that people become more definitive when thinking in the long term, while randomness/uncertainty increases with the reduction of the planning period (time budget).

The constant term in the baseline utility component indicates the reference baseline utility (if all variables in the baseline utility component are considered to be zero). This component is the highest for Sunday compared to the other days of the week. The high reference utility indicates the lack of sufficient information for Sunday compared to the other days.

The translating parameter, $\theta$ gives the non-zero reference for the additional utility component and ensures the possibility of zero participation in any activity types. The value of this parameter is the lowest for Monday and increases with the weekdays and again reduces at the end (Sunday). The higher value of this parameter basically indicates the higher possibility of zero participation in some activities but higher number of total activity participations. The higher value for the days at the middle of the week indicates that we usually participate in a higher number of activities mid-week, but these tend to be concentrated in certain activity types, while other activities tend not to be engaged in at all mid-week. It also indicates serial nonparticipation in some activities during the middle of the week. Whereas, the lower values at the beginning or the end of the week indicate we tend to participate in variety of activities.

The $\rho$ parameter indicates the sensitivity of the effect of the composite activity on activity program generation. The value of $1-\exp (\rho)$ is higher for the starting day of the week compared to the other days. It indicates that we are more cautious for allocating time to the composite activity at the beginning of the week but eventually the time pressure increases and we become less 
concerned about it. This finding is very important and it also reinforces our argument in favour of week-long modelling as opposed to typical-day modelling. This finding also ensures that our models comply with the behavioural psychology of time allocation and activity/travel behaviour (Gärling et al. 1999).

In terms of the variables in the baseline utility component: age of the person is significant for earlier days of the week, indicating that older people plan for higher numbers of activities than younger people. Although the dummy variable representing sex did not show a significant parameter, the values and signs indicate that males plan for higher numbers of activities in the middle of the week but females plan for higher numbers of activities in the last day of the weekend (Sunday) and the first day of the weekdays (Monday). Income becomes significant for the last day of the weekdays (Friday) and it shows negative effect. It indicates higher income people plan for lower number of activities on Friday compared to lower income people. Higher household automobile ownership and possession of driving license levels give people higher modal accessibility and influence them to plan more activities. Intuitively this finding is justified and these variables help to link the activity program generation model with the other components of an integrated model of short-, medium- and long-term household decisions (e.g. an auto ownership model) in a meaningful way. The total working hours of the workers, which is a skeleton activity component (Habib \& Miller 2006c) is highly significant in every day-specific model. This variable shows a similar negative effect in every model except for the whole week model, where the sign is opposite but statistically insignificant. This implies that this variable captures the within-day dynamics in time allocation behaviour. The work activity is fundamentally a different type of activity. People earn money by participating in work activity, whereas they usually spend money for participation in other activities. The negative sign of this 
variable indicates that the work activity acts as a temporal peg, not only in the case of activity scheduling, but also at the activity program generation level. If people spend more time in working, they allocate less time to other activities, rather than reducing time from the composite activity. Whereas in the case of the whole week aggregate model, the interpretation of the positive sign of this variable is that people who plan larger amounts of time for work throughout the week allocate lower amounts of time for composite activities rather than reducing time from the other activities. It also implies that the aggregate weekly model fails to capture the withinday dynamics in time-use and activity planning behaviour.

The activity specific dummy variable in the baseline utility component gives significant insight into the behavioural process. In this case the absolute values and signs of the coefficients do not have any practical significance, but the relative values indicate the corresponding importance of individual activities in agenda formation. In other words, these variables can be interpreted as relative direct utility gain or relative direct satisfaction or relative preference. It is clear that the least preferred activity is Act 03, which is the drop off/pick up goods activity. It is intuitive that the drop off/pick up goods type of activities may not give direct utility per se but these activities provide necessary support for other activities. Act 15 (volunteer activities) also seems to be less preferred in comparison to other activities. The most preferred activity is Act 10, which is at-home recreation type activities. This type of activity does not require travel, and people enjoy the process of the activities too. Act 02 also has higher relative importantance. Act 02 is the household obligation type activity, and it seems that people get high satisfaction by performing this type of activities. Among the three shopping activities (Act 04, Act 05 and Act 06) the personal clothing and other type of shopping (Act 06) gives higher relative satisfaction compared to the other two shopping types, an intuitively justified result. Between the two types 
of social activities, Act 11 and Act 12, the visiting and hosting type (Act 11) social activities show higher satisfaction then the religious or cultural type social activities (Act 12). The relative direct utility from activities involving ICT (Information and Communication Technology), Act 14, has lower direct utility than direct interactive social activities (Act 11 and Act 12) and recreation activities (Act 09 and Act 10), but online shopping (Act 14) has higher direct utility than major grocery type shopping activity (Act 04). It should be noted that these behavioural findings are consistent in all models shown in Table 1.

The variables in the additional utility component are key variables and they define the attraction/detraction potential of the activities under consideration. The variable 'travel ratio', which can be considered as the time cost of the activity has a negative effect in all models. Intuitively it is sensible that an activity that involves longer travel time reduces the relative interest in that activity compared to others that involve lower travel time. But it is interesting to note that this variable becomes statistically insignificant for the case of Sunday. The justification is that the travel time often becomes an insignificant factor for activity planning in the weekends. The difference between Sunday and Saturday is that Saturday is the beginning of the weekend and it may carry the time pressure developed through out the week and hence, travel time at that case does affect significantly in activity planning. The variable "number of possible activity locations' also shows a negative effect. It indicates that the higher the number of possible locations the person has for any activity; the lower the frequency for that activity.

The variable indicating the total number of executed activities of each activity type under consideration is highly significant in every day-specific model. This is a key variable that defines the day-to-day dynamics in time-use and activity/travel behaviour. It also introduces the dynamic linkage between activity scheduling and activity program generation. The sign of this variable is 
positive. It appears that the positive sign captures the repeated participation in specific activities or serial non-participation in certain activities. As indicated in Figure 1, the maximum observed frequency of some activities are very high compared to some other activities, and in practical life our all activities are not uniformly distributed throughout the week.

\section{Conclusions}

This paper describes a day-specific activity program generation model for week-long activity/travel scheduling. Considering the daily time budget, the models use the Kuhn-Tucker optimality conditions to derive the optimum set of activities (including unspecified composite activities) within the budgeted time period. The models capture the within-day dynamics of timeuse and activity/travel behaviour by incorporating 'total working hours' in the model and day-today dynamics are accommodated considering 'previous day's total executed activities' as variables. These two types of variables also make the activity program generation models sensitive to the activity scheduling process. The incorporation of the variable, 'travel ratio' introduces the transportation system performance inside the activity program generation model. A number of other socio-economic and activity-specific variables give insight into the behavioural process of time-use and activity/travel behaviour. The models are estimated using 2002-2003 CHASE survey data collected in Toronto. The models show significant goodness of fit to the observed data. Significant variations in behaviour are obvious throughout the week. The starting (Monday) and ending (Sunday) of the week are distinctively different from the other days in terms of goodness of fit to the observed data, number of statistically significant variables, the effects of travel time in activity/travel planning etc. The models also replicate our repeated participation and serially non-participation in some activities. 
In terms of application, the models described in this paper are part of the continuing effort to make the activity-based travel demand model more behavioural and more sensitive to various land-use and transportation policies. This paper compliments other work of the authors, in which a daily activity skeleton was derived (Habib \& Miller 2006c). All of this research is leading to the development of 'second generation' version of the existing activity-based travel model TASHA (Miller \& Roorda 2003).

\section{Acknowledgement}

This research was supported by a Transport Canada TPMI grant, as well as financial contributions by the Neptis Foundation, EJLB Foundation, McLean Foundation, Salamander Foundation, and the Greater Toronto Airports Authority. The data used in the study were collected as part of a Canada SSHRC MCRI grant. Authors also acknowledge the comments of five anonymous referees.

\section{References}

Aptech Systems (2006) GAUSS User's Manual. Maple Valley, CA.

Arentze T \& Timmermans HJP (2005) ALBATROSS Version 2.0 A Learning Based Transportation Oriented Simulated System, EIRASS.

Arora N, Allenby GM \& Ginter JL (1998) A hierarchical Bayes model of primary and secondary demand. Marketing Science 17(1): 29-44.

Bhat CR \& Koppelman FS (1993) A conceptual framework of individual activity program generation. Transportation Research A 27(6): 433-446.

Bhat CR (2005) A multiple discrete-continuous extreme value model: Formulation and application to discretionary time-use decisions. Transportation Research Record Part B 39: 679707. 
Bhat CR, Guo JY, Srinivasan S \& Sivakumar A (2004) A Comprehensive econometric microsimulator for daily activity travel patterns (CEMDAP). Paper presented at $83^{\text {rd }}$ Annual Meeting of the Transportation Research Board, Washington, D. C.

Doherty ST \& Miller EJ (2000) A computerized household activity scheduling survey. Transportation 21(1): 75-97.

Doherty ST (2006) Should we abandon activity type analysis? Redefining activities by their salient features. Transportation (Forthcoming).

Doherty ST, Nemeth E, Roorda MJ \& Miller EJ (2004) Design and assessment of Toronto area computerized household activity scheduling survey. In Transportation Research Record: Journal of the Transportation Research Board 1894, TRB, National Research Council, Washington DC, 140-149.

Ettema D (2005) Latent activities: Modelling the relationship between travel times and activity participations. In Transportation Research Record: Journal of the Transportation Research Board 1926, TRB, National Research Council, Washington DC, 171-181.

Gärling T, Brännäs K, Garvill J, Golledge RG, Gopal S, Holm E \& Lindberg E (1989) Household activity scheduling. In: Transportation Policy, Management and Technology Towards 2001: Selected Proceedings of Fifth World Conference of Transportation Research IV: 235-248, Ventura, CA: Western Periodical.

Gärling T, Gillholm R \& Montgomery W (1999) The role of anticipated time pressure in activity scheduling. Transportation 26: 173-191.

Gärling T, Kwan M -P \& Golledge RG (1994) Computation-process modelling of household activity scheduling. Transportation Research B 28(5): 355-364.

Gärling, T., and J. Garvill. Psychological explanation of participation in everyday activities. In: Behaviour and Environment: Psychological and Geographical Approach (T. Gärling and R. G. Golledge, ed.), Amsterdam, New York: Elsevier Science Publisher, 1993

Habib KMN \& Miller EJ (2005) A conceptual model of activity-based travel demand model. In: Proceedings of PROCESSUS Second International Colloquium on the Behavioural Foundation of Land-Use Transportation Model: Frameworks, Models and Applications, June 12-15, Toronto.

Habib KMN \& Miller EJ (2006a) Modelling activity generation: A utility based model for activity agenda formation. Paper presented at the $11^{\text {th }}$ International Conference on Travel Behaviour Research, Kyoto.

Habib KMN \& Miller EJ (2006b) Modelling individuals' frequency and time allocation behaviour for shopping activities considering household level random effects. Transportation Research Record (Forthcoming) 
Habib KMN \& Miller EJ (2006c) Modelling skeleton components of workers' daily activity schedules. Transportation Research Record (Forthcoming).

Kitamura R, Yamamato T, Susilo YO \& Axhausen KW (2003) On the day-to-day variability of prism vertex location. Paper presented at $82^{\text {nd }}$ Annual Meeting of the Transportation Research Board, Washington, DC.

Kuhn HW \& Tucker AW (1951) Nonlinear programming. In: Neyman J (eds) Proceedings of the Second Berkeley Symposium on Mathematical Statistics and Probability: 481-492, University of California Press, Berkeley, CA.

Litwin M (2005) Dynamic Household Activity Scheduling Process. Ph.D Thesis, Department of Civil Engineering, University of Toronto.

Miller E J \& Roorda MJ (2003) Prototype model of household activity/travel scheduling (TASHA). In Transportation Research Record: Journal of the Transportation Research Board, No. 1831, TRB, National Research Council, Washington, DC, 114-121.

Miller EJ (2005) Project-based activity scheduling for household and person agents. In Mahmassani H (eds) Flow, Dynamics and Human Interactions-Proceedings of the $16^{\text {th }}$ International Symposium on Transportation and Traffic Theory, Elsevier: New York.

Pendyala R, Kitamura R, Kikuchi A, Yamamato T \& Fujii S (2004) FAMOS: the Florida activity mobility simulator. Paper presented at $83^{\text {rd }}$ Annual Meeting of the Transportation Research Board, Washington, DC.

Roorda MJ \& Miller EJ (2004) Toronto area panel survey: Demonstrating the benefits of a multiple instrument panel survey. Preprint CD of Seventh International Conference on Travel Methods, Costa Rica, August 1-2.

Roorda MJ \& Ruitz T (2006) Long and short term dynamics in activity scheduling: A structural equation approach. Paper presented at the $11^{\text {th }}$ International Conference on Travel Behaviour Research, Kyoto, August.

Salvini P \& Miller EJ (2005) ILUTE: An operational prototype of comprehensive microsimulation model of urban system. Network and Spatial Econometrics 5: 217-234. Schlich R \& Axhausen KW (2003) Habitual travel behaviour: evidence from a six-week travel diary. Transportation 30: 13-36.

von Haefen RH \& Phaneuf DJ (2004) Kuhn-Tucker demand system approach to non-market valuation. In: Scrapa R \& Alberini A (eds) Applications of Simulation Methods in Environment and Resource Economics, Kluwer Academic Publishers, Boston/Dordetch/London.

Winston GC (1987) Activity choice: A new approach to economic behaviour. Journal of Economic Behaviour and Organization 8: 567-588. 
About the authors

Khandker M. Nurul Habib, PhD

Eric J. Miller, PhD

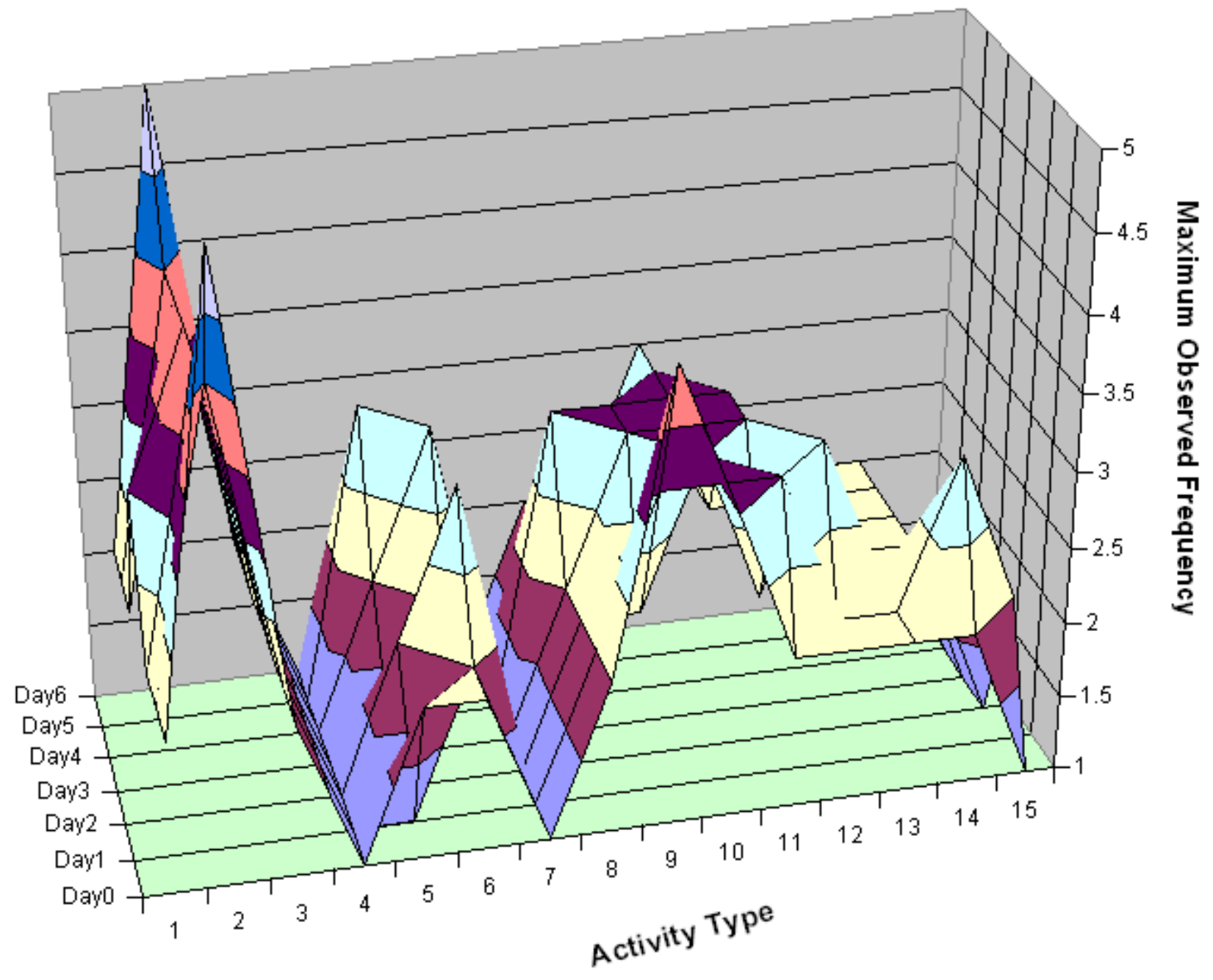


Figure 1: Observed Maximum Frequencies of the Activities 
Table 1: Kuhn-Tucker Models for Activity Program Generation

\begin{tabular}{|c|c|c|c|c|c|c|c|c|}
\hline & Monday & Tuesday & Wednesday & Thursday & Friday & Saturday & Sunday & Whole Week \\
\hline Parameters & Values( $|t|)$ & "Values( $|t|)$ & "Values( $|t|)$ & "Values( $|t|)$ & "Values( $|t|)$ & "Values( $|t|)$ & Values( $|t|)$ & Values( $|t|)$ \\
\hline Scale Parameter & $-0.08(2.79)$ & $-0.03(1.22)$ & $-0.06(2.23)$ & $-0.04(1.60)$ & $-0.02(0.66)$ & $-0.04(1.33)$ & $0.002(0.06)$ & $-0.16(5.07)$ \\
\hline \multicolumn{9}{|l|}{ Baseline Utility Component } \\
\hline Constant & $-11.7(10.39)$ & $-13.2(11.19)$ & $-12.3(10.4)$ & $-12.44(10.7)$ & $-13.7(11.85)$ & $-13.1(10.15)$ & $-14.1(8.93)$ & $-11.81(7.45)$ \\
\hline Age in 10 years & $0.005(2.08)$ & $0.007(2.52)$ & $0.007(2.35)$ & $0.002(0.83)$ & $0.007(2.44)$ & $0.005(1.89)$ & $0.003(0.81)$ & $0.003(1.58)$ \\
\hline Male (Dummy) & $-0.08(1.14)$ & $0.09(1.3)$ & $0.08(1.11)$ & $0.06(0.92)$ & $0.08(1.05)$ & $0.11(1.56)$ & $-0.11(1.35)$ & $-0.07(1.42)$ \\
\hline Income in $1000 \mathrm{CAD} /$ Year & $6 \mathrm{E}-4(0.68)$ & $-3 E-4(0.29)$ & $4 \mathrm{E}-4(0.44)$ & $12 \mathrm{E}-3(1.17)$ & $-17 \mathrm{E} 3(1.72)$ & $-11 \mathrm{E}-3(1.05)$ & $1 \mathrm{E}-4(0.05)$ & $8 \mathrm{E}-4(1.22)$ \\
\hline Driving License (Dummy) & $0.10(0.80)$ & $0.13(1.04)$ & $0.16(1.28)$ & $0.23(1.51)$ & $0.29(1.93)$ & $0.27(2.24)$ & $0.08(0.58)$ & $0.14(1.68)$ \\
\hline Household Size & $0.12(1.35)$ & $-0.04(0.43)$ & $0.05(0.52)$ & $0.08(0.80)$ & $-0.04(0.31)$ & $-0.14(1.48)$ & $0.12(0.94)$ & $-0.02(0.35)$ \\
\hline $\begin{array}{l}\text { Household Autos }>2 \\
\text { (Dummy) }\end{array}$ & $0.03(0.21)$ & $0.08(0.58)$ & $0.34(2.55)$ & $-0.02(0.10)$ & $0.18(0.92)$ & $0.23(1.56)$ & $0.09(0.63)$ & $0.02(0.28)$ \\
\hline Total Working Hours & $-0.04(4.31)$ & $-0.04(4.77)$ & $-0.03(3.28)$ & $-0.04(3.77)$ & $-0.03(2.73)$ & $-0.02(2.33)$ & $-0.03(2.60)$ & $0.002(1.35)$ \\
\hline \multicolumn{9}{|l|}{ Activity Specific Dummy } \\
\hline ActType01 & $1.44(6.97)$ & $1.07(5.27)$ & $1.00(5.42)$ & $1.31(3.67)$ & $1.18(3.15)$ & $1.01(4.51)$ & $1.07(5.16)$ & $1.15(3.17)$ \\
\hline ActType02 & $2.62(12.06)$ & $2.49(14.22)$ & $2.38(13.03)$ & $2.53(7.07)$ & $2.43(6.68)$ & $2.50(11.54)$ & $2.46(13.28)$ & $2.50(6.53)$ \\
\hline ActType 03 & $-0.32(1.29)$ & $-0.18(0.91)$ & $-0.10(0.54)$ & $-0.26(0.72)$ & $-0.30(0.80)$ & $-0.20(0.79)$ & $-0.20(0.85)$ & $-0.24(0.70)$ \\
\hline ActType04 & $0.61(2.87)$ & $0.51(2.42)$ & $0.48(2.52)$ & $0.21(0.53)$ & $0.46(1.13)$ & $0.19(0.72)$ & $0.34(1.41)$ & $0.62(1.61)$ \\
\hline ActType05 & $0.37(1.69)$ & $-0.06(0.52)$ & $0.29(1.79)$ & $-0.04(0.12)$ & $-0.30(0.82)$ & $-0.01(0.17)$ & $-0.23(0.89)$ & $0.14(0.41)$ \\
\hline ActType06 & $1.06(4.83)$ & $0.86(4.19)$ & $1.08(6.34)$ & $1.02(2.77)$ & $0.77(2.01)$ & $0.95(4.24)$ & $0.80(3.70)$ & $1.25(3.40)$ \\
\hline ActType07 & $0.19(0.87)$ & $-0.19(0.87)$ & $0.30(1.37)$ & $-0.44(0.92)$ & $0.06(0.14)$ & $-0.04(0.12)$ & $0.26(0.98)$ & $0.08(0.21)$ \\
\hline ActType08 & $0.19(0.87)$ & $0.26(1.18)$ & $0.23(1.29)$ & $0.25(0.62)$ & $-0.08(0.43)$ & $0.27(1.25)$ & $0.01(0.28)$ & $0.43(1.21)$ \\
\hline ActType09 & $2.27(10.34)$ & $2.22(12.22)$ & $1.96(10.96)$ & $2.31(6.50)$ & $2.28(6.28)$ & $2.07(9.34)$ & $2.07(11.15)$ & $2.06(5.40)$ \\
\hline ActType10 & $3.13(14.26)$ & $3.02(17.13)$ & $2.93(15.93)$ & $3.08(8.44)$ & $2.93(8.08)$ & $2.95(13.82)$ & $3.05(17.13)$ & $3.19(8.35)$ \\
\hline ActType11 & $2.10(10.01)$ & $2.34(12.09)$ & $2.04(11.53)$ & $2.21(6.08)$ & $2.09(5.74)$ & $2.26(9.69)$ & $1.89(7.78)$ & $2.33(6.60)$ \\
\hline ActType12 & $1.05(4.66)$ & $0.93(4.01)$ & $1.33(6.91)$ & $1.18(3.16)$ & $1.09(2.85)$ & $1.07(4.31)$ & $0.89(3.86)$ & $2.33(2.43)$ \\
\hline ActType13 & $1.08(5.13)$ & $1.20(5.96)$ & $1.12(7.87)$ & $1.17(3.13)$ & $0.96(2.71)$ & $1.16(5.32)$ & $0.92(4.04)$ & $1.20(3.68)$ \\
\hline ActType14 & $1.27(5.80)$ & $1.31(7.62)$ & $1.24(6.70)$ & $1.47(3.99)$ & $1.37(3.78)$ & $1.34(6.17)$ & $1.37(7.06)$ & $1.04(2.65)$ \\
\hline ActType15 & $0.21(0.85)$ & $-0.08(0.23)$ & $0.37(1.29)$ & $0.51(1.13)$ & $0.33(0.69)$ & $0.34(0.93)$ & $0.17(0.48)$ & $-0.07(0.15)$ \\
\hline \multicolumn{9}{|l|}{ "Translating Parameter } \\
\hline Theta & $-0.41(4.39)$ & $-0.37(3.95)$ & $0.08(0.55)$ & $0.10(0.92)$ & $0.14(1.60)$ & $-0.14(1.46)$ & $0.31(4.72)$ & $-0.32(2.15)$ \\
\hline
\end{tabular}

Additional Utility 


\begin{tabular}{|c|c|c|c|c|c|c|c|c|}
\hline \multicolumn{9}{|l|}{ Component } \\
\hline Travel Ratio & $-0.23(3.01)$ & $-0.48(7.23)$ & $-0.22(1.96)$ & $-0.22(2.72)$ & $-0.20(3.34)$ & $-0.33(4.79)$ & $-0.03(0.88)$ & $-0.34(3.67)$ \\
\hline $\begin{array}{l}\text { Number of Possible } \\
\text { Locations For the Activity }\end{array}$ & $-0.03(1.84)$ & $-0.02(1.55)$ & $-0.01(0.90)$ & $-0.01(0.63)$ & $0.00(0.44)$ & $-0.03(2.59)$ & $-0.01(0.55)$ & $-0.01(0.86)$ \\
\hline \multicolumn{2}{|l|}{$\begin{array}{l}\text { Previous Day Number of } \\
\text { Executed Activities }\end{array}$} & $0.69(10.64)$ & $0.88(12.98)$ & $0.95(14.91)$ & $0.83(14.86)$ & $0.81(13.24)$ & $0.95(13.13)$ & \\
\hline \multicolumn{9}{|c|}{ Composite Good Parameter } \\
\hline Rho & $0.69(8.34)$ & $0.82(10.45)$ & $0.74(8.17)$ & $0.75(8.69)$ & $0.82(10.75)$ & $0.79(8.61)$ & $0.87(8.39)$ & $0.55(5.26)$ \\
\hline Pseudo-R square & 0.177 & 0.220 & 0.200 & 0.225 & 0.204 & 0.217 & 0.197 & 0.177 \\
\hline
\end{tabular}

